\title{
PSICÓLOGOS Y PSICÓLOGOS
}

\author{
Jullo Seoane \\ Universidad de Valencia
}

\section{RESUMEN}

La psicologla, como otros campos de conocimiento ofrece pocos datos a favor de un modelo histórico acumulativo y unitario. En su lugar, parece que representa un considerable volumen de conocimientos socioculturalmente determinados (esto implica a su vez, temporalmente determinados). Dos tipos de psicologia (la sanitania y la solidaria) aglutinan a la mayor parte de pensamiento y acción psicológica y el presente real es el de un conglomerado de modelos, técnicas y procedimientos de imposible unidad que caracterizan el quehacer del psicólogo; y todos ellos parecen necesarios.

Palabras clave: PSICOLOGIA Y SOCIEDAD, UNIDAD DE LA PSICOLOGIA, PROFESIONALIZACIÓN CULTURALMENTE IMPUESTA.

\section{SUMMARY}

Psychology like others fields of knowledge, offers little data in favour of only one scientific and historical accumulative model for the development of scientific knowledge. It is more similar to a 
conglomerate volume of facts, models and procedures determined by social and cultural forces (and time dependent). Health psychology and solidarity psychology are proposed as general lines of thought, methodology and practice. All models, procedures and practices seem necessaries, and subsequentty, all psychologists types must be in existence.

Key words: PSYCHOLOGY AND SOCIETY, UNITY OF PSYCHOLOGY, CULTURAL INFLUENCES ON PROFESSION OF PSYCHOLOGIST.

Debo de agradecer al Prof. Pelechano la invitación a participar en este colectivo sobre la formación del psicólogo y sobre la enseñanza de la propia psicologla. Supongo que, además de la amabilidad, su petición estaba condicionada por toda una serie de recuerdos de hace ya vanias décadas, cuando en distintas ocasiones estuvimos encerrados durante tardes enteras en algún centro oficial de Madrid, junto con Mariano Yela, José Luís Pinillos y otros más, con el propósito de construir un plan de psicología, una programación de la carrera o simplemente un listado completo de las disciplinas necesarias para la formación del psicólogo. Y también discutiendo sobre lo mismo a altas horas de la madrugada, después de un congreso profesional, en algún hotel de Santiago de Compostela, Vigo, Murcia, Alicante o Barcelona. Creo recordar que nunca estuvimos completamente de acuerdo, pero eso era lo menos importante, entre otras cosas porque los distintos proyectos que realizábamos nunca aparecían tal cual en los papeles oficiales, aunque siempre hacíamos improbos esfuerzos por reconocernos en ellos. Seguramente era la "mano invisible" de Adam Smith la que tomaba las decisiones finales 0 , con mayor probabilidad, alguna voz invisible que dictaba las órdenes oportunas después de pedir nuestra opinión. O quizás antes de pedirla.

En cualquier caso, bien está lo que nunca acaba, porque una vez más estamos ante los mismos problemas. ¿Qué debe saber un profesional de la psicología? ¿Cuál es el bagaje intelectual de un buen docente de nuestra disciplina? La primera conclusión es evidente después de tantos intentos más o menos afortunados, pero 
siempre desbancados por el siguiente proyecto y la próxima planificación. $O$ bien es un problema sin solución, en cuyo caso hay que poner aqul un punto final, mirar hacia otro lado y seguir trabajando en cosas más productivas. $O$ bien los psicólogos y sus maestros necesitan una preparación distinta en cada momento, diferentes programas y habilidades, en función de la época, la cultura y las necesidades sociales. Aunque solo sea por disciplina metodológica, amén de justificación personal, me inclino por la segunda opción. No existen planes excelentes ni conocimientos definitivos, solo es posible establecer una relación entre lo que se debe saber y las características de una determinada época social. Esa relación puede adoptar muchas formas, puede ser inteligente, torpe, mecánica, reduccionista, imitativa o mercantilista, entre otras muchas, pero en los matices de esa interacción radica todo el problema. Precisamente sobre eso me gustaria hablar en esta ocasión.

\section{PLANTEAMIENTO Y TESIS}

Para empezar, es conveniente no engañar a nadie. En todo lo que sigue no aparecerá en ningún momento un plan de estudios acabado, ni un listado de especialidades y mucho menos una geografía de las áreas de conocimiento psicológico. Cada tarea tiene su momento y esa ya no me corresponde ahora, cuando existen muchos otros mejor preparados y más motivados para realizar esa labor. En esta ocasión, prefiero arroparme en los consejos de Nietzsche sobre la materia, cuando advierte al lector: "Por último, no debe, al final, a modo de resultado, esperar nuevos cuadros sinópticos y nuevos programas para los colegios, ni para otros establecimientos de enseñanza". La afición por los cuadros sinópticos habría que traducirla ahora por la adicción a los gráficos de PowerPoint, tan útiles en muchos momentos pero fuera de lugar para el objetivo principal de estas páginas.

Casi siempre que alguien se enfrenta a la formación de profesionales y docentes, tiene como telón de fondo un supuesto de base que condiciona fatalmente su tarea. Supone sin duda que la psicología que merece ser enseñada y practicada nació, al menos con- 
vencionalmente, hace poco más de un siglo y desde entonces se desarrolla con una tendencia lineal, acumulativa y aumentando en perfección. Es decir, que progresa adecuadamente. De aquí se deducen inevitablemente tres consecuencias para la tarea de la formación actual. En primer lugar, las investigaciones y publicaciones de última hora son las mejores, en términos generales, de todos los tiempos pasados; $y$, además, constituyen el resumen de todo el conocimiento anterior. Son estas aportaciones recientes las únicas que merecen ser estudiadas y enseñadas, las anteriores solo tienen cierto interés didáctico, como material de entrenamiento para los novicios o para contraponerlas al progreso conseguido en la actualidad.

En segundo lugar, si lo último es lo mejor, el problema de la formación y práctica consiste en poner orden en todo este conocimiento ya conseguido, que resulta demasiado extenso y diversificado para comprimirlo en unos pocos años de estudio. Es aquí donde entran las perspectivas docentes, los cuadros sinópticos, en función de los intereses de grupos, expectativas de investigación y salidas profesionales. La diversidad de programas y especialidades propuestos en los diversos planes es la que proporciona un aspecto de actividad y dinámica progresiva en el conocimiento del momento. El punto de vista, la perspectiva, la rotación factorial aparenta nueva creación teórica, que se entiende como un grado más del progreso imparable de la psicología cientifica.

Por último, los conocimientos alcanzados tienen que producir innovaciones tecnologicas, instrumentos construidos con los últimos adelantos que justifican el progreso conseguido. De ahí la urgencia para generar nuevos instrumentos, para concentrar los recursos de investigación en la construcción de herramientas con las que deben entrenarse los profesionales como mensajeros de los últimos adelantos. Aparecen así con ritmo vertiginoso nuevas terapias, pruebas psicológicas, diagnósticos oniginales, técnicas educativas, aplicaciones insolitas, todo un amplio repertorio que intenta garantizar el conocimiento psicológico en función del reconocimiento social.

Es posible que ese modelo de psicologla haya sido el adecuado durante todo este tiempo. Es más, si se pudo defender con cierta dignidad hasta ahora es porque fue conveniente para una época 
social de considerable duración. Sin embargo, los modelos y los conceptos no duran eternamente, necesitan cambiar y adaptarse a las nuevas circunstancias a las que pertenecen. El progreso es relativo y afecta a una determinada teorfa, a una concepción o a un grupo de técnicas concretas. La construcción acumulativa de conocimiento no puede tener un sentido absoluto, está condicionada por épocas y circunstancias. En resumen, las cosechas pueden ser ricas y abundantes, pero las estaciones varían y los terrenos se agotan, hasta el planteamiento ecológico más elemental sabe que los recursos no son inagotables.

Resulta curioso que todo el mundo acepte con facilidad que la sociedad actual tiene características radicalmente nuevas, destacando cada uno el aspecto que más le sorprende dentro del amplio abanico existente $y$, sin embargo, continuamos construyendo la misma psicologia de siempre, con las mismas puertas y ventanas, al margen de algunos retoques omamentales o ligeros cambios en el decorado. Puede que sea necesario replantearse lo que investigamos, también lo que enseñamos $y$, por supuesto, las prácticas profesionales en función de la nueva sociedad que nos rodea. Será sin duda una labor difícil y compleja, el producto de presiones más externas que de reconocimiento interno, pero mientras tanto podemos señalar ya algunos aspectos que están afectando a la psicologia y modíficando buena parte de su fisionomía.

\section{COMUNICACIÓN, CONSENSO Y PARTICIPACIÓN}

La psicología surgío y se desarrolló inicialmente en una sociedad muy distinta a la actual, en una época donde las diferencias culturales estaban muy marcadas y las posibilidades de información eran escasas cornparadas con las que tenemos hoy en día. Sus planteamientos e investigaciones estaban restringidos a círculos muy cerrados, principalmente filósofos, médicos y pensadores sociales, de forma que sus aportaciones no tenían una repercusión inmediata ni en sectores muy amplios de la sociedad. Esta situación contrasta radicalmente con lo que ocurre en nuestros días, puesto que la actividad de la psicologia en todos sus planos está en contacto $\theta$ 
interacción continua con la sociedad en su sentido más amplio. Analizar el sentido de los cambios ocurridos en los últimos doscientos años y su repercusión en nuestra disciplina está fuera de lugar en este escrito, pero se podrían resumir las principales tendencias comentando brevemente las nuevas características que se han producido en la cultura social, científica y profesional de la actividad psicológica.

\section{a) Cultura soclal}

En cuanto a la cultura social que rodea a la psicología, es necesario reconocer que está marcada especialmente por la comunicación. Me refiero a que no existe ninguna otra disciplina de las llamadas científicas que esté más identificada con todo tipo de medios de comunicación, más en contacto con la divulgación de sus conocimientos o pendiente de las expectativas sociales. Pero en las sociedades abiertas, la comunicación tiene dos direcciones y a medida que la psicología penetraba más en la sociedad, las creencias, expectativas y deseos de la sociedad sobre lo que espera de la psicología influyen en el desarrollo de nuestra disciplina. Los trabajos de Wundt se distribuyeron lentamente por los caminos estrechos de la comunicación científica, mientras que la "inteligencia emocional" solo necesitó un libro y pocos meses para desencadenar unas expectativas que se transformaron en líneas de investigación, tesis doctorales y seguidores convencidos. Sería absurdo defender actualmente el ritmo de difusión de las teorías de Wundt, pero también resulta inadmisible no reconocer sinceramente la presión social sobre el desarrollo de la disciplina.

Hace unas cuantas décadas, algunos pusieron de moda el dicho de que la psicología era lo que hacian los psicólogos. Tenía poco sentido decirlo entonces, pero mucho menos ahora cuando es la sociedad completa la que elabora los contenidos y desarrollos, al margen de que los psicólogos ocupen una posición privilegiada, como expertos, en esa elaboración. Los índices de impacto de nuestras publicaciones son realmente índices de audiencia, que se transforman automáticamente en recursos para investigar, privile- 
gios para ocupar puestos y divulgación en los medios de comunicación. Es posible que esta tesis pueda parecer demasiado ligera, pero sería muy fácil demostrarla comparando las principales líneas de investigación de centros y universidades con los titulares más importantes de la prensa o con las preocupaciones ciudadanas en las encuestas de opinión pública.

\section{b) Cultura clentíflca}

Por otra parte, la cultura científica también tiene novedades importantes. No me refiero especialmente a los debates propios de la metodología científica o, en general, a la filosofía de la ciencia. Me refiero al conjunto de creencias y sentimientos de la sociedad relacionados con el conocimiento científico, a la tendencia de interpretar la actividad tradicional de la ciencia como un ritual de comportamientos anticuados y excesivamente aparatosos para los tiempos modernos. La necesidad de consenso rompe la vieja liturgia de la ciencia en mil pedazos. En un escrito anterior, hace algunos años, manifesté la misma opinión que ahora y que reproduzco a continuación.

Cuando el científico deja de ser un romántico solitario y se introduce en las grandes organizaciones de investigación, la ciencia se convierte en un servicio más, como la sanidad o la educación, y el científico se transforma en un técnico o experto de ese sector. Al sustituir al científico clásico por el experto, se acorta la distancia con el lego, con el ciudadano corriente y es entonces cuando surge la firme convicción de que cualquiera, con un mínimo esfuerzo, se puede convertir en experto. De hecho, los ciudadanos que se ven afectados por alguna catástrofe colectiva, una situación de emergencia o alguna dolencia, se convierten en poco tiempo en expertos de ese fenómeno o dolencia. En psicología ocurre lo mismo y posiblemente con mayor facilidad porque, aunque se continúa defendiendo su carácter científico, se acepta abiertamente la pluralidad metodológica, la equivalencia funcional de procedimientos, que se manifiesta en una indiferencia hacia los medios con tal de que alcancen la solución deseada; y, además, se concede con rapidez 
el título de experto, en parte debido al incremento de las destrezas culturales y del acceso a la información en las nuevas sociedades, donde el simple propósito de ayudar a los demás, de actuar en los servicios sociales o de pertenecer a alguna organización humanitaria convierte al lego en experto en psicología o en alguna técnica concreta de nuestra disciplina. La validez del conocimiento psicológico ya no radica en la metodología (ciencia clásica) ni tampoco en la comunidad científica (paradigma kuhniano), sino en el consenso entre expertos, ciudadanos y afectados.

Esa es la razón por la que el acuerdo producido por consentimiento entre distintos grupos se convierta en la actualidad en una de las bases más sólidas de la actividad científica. Conferencias de consenso para determinar tipos de diagnóstico o de tratamientos especificos, conferencias de decanos, de rectores, de expertos, de estudiantes para estructurar los estudios, acuerdos en el Espacio Europeo para la Educación Superior, siempre la generación de consenso como uno de los fundamentos de la actividad y de la producción científica. Esta tendencia tiene hasta conceptos y desarrollos propios, que ahora no vienen al caso, como por ejemplo la transdisciplinariedad o también el Modo 2 de producción del conocimiento, que es aquel que se produce en constante interacción con otros grupos sociales ajenos en principio a la producción científica.

\section{c) Cultura profeslonal}

Por último, la cultura profesiona/ dentro del ámbito de la psicología actual tiene características y sensibilidades bastante distintas a las de hace pocas décadas, cuando lo importante para un profesional era cultivar tres aspectos de su trabajo: la sólida base del rigor metodológico para ordenar su actividad, el respaldo de un reconocido marco té́rico para garantizar sus interpretaciones y el amparo de la academia o de alguna institución que proporcione credibilidad a la formación adquirida.

Es evidente, además de los comentarios anteriores, que estas características son virtudes un poco antiguas que ya no son valoradas de la misma manera. Abandonados por el método, aplastados 
por la teoría y demorados excesivamente por la formación, la mejor solución consiste en fomentar grandes reuniones de profesionales, ya sea por especialidades o simplemente por áreas geográficas de influencia, donde el prestigio profesional no proviene de la propia actividad técnica sino de la participación continuada y sistemática en todo tipo de congresos y reuniones, a lo largo de todo el mundo, en los que se generan grandes masas de información sobre la actividad profesional y que alimentan posteriormente todos los sistemas de comunicación. En consecuencia, el reconocimiento proviene de la participación en todos los circuitos posibles de reuniones profesionales, de los índices de impacto en los sistemas de comunicación y de colaborar en el consenso alcanzado por todos en los diversos temas profesionales.

Comunicación, consenso y participación se convierten en las actividades de éxito profesional, al margen del contenido específico de la propia actividad. Puede suponerse, por tanto, que las viejas instituciones de formación y de intercambio profesional, como por ejemplo la Universidad, quedan bastante alejadas de sus funciones originales, utilizándose ahora exclusivamente como «servidores" de las redes de información profesional. En esto como en otras muchas cosas, la psicologla no es diferente de las demás disciplinas de su entorno, donde ya ni siquiera es cierto que la práctica profesional sea el fundamento más firme de la producción de teorías, sino que se limita a navegar por los sistemas de comunicación intercambiando información y experiencias personales.

Tanto el profesional como el docente de la psicología deberán saber una serie de conceptos y terminología propia de su actividad, pero lo más evidente es que tendrán que desarrollar habilidades especiales para moverse con fluidez en la sociedad actual, vale decir, entre los medios y sistemas de comunicación, habilidades también para contribuir en la generación de consenso en el conocimiento de su especialidad $y$, desde luego, participar con frecuencia en las reuniones y congresos repartidos a lo largo y ancho de la geografía psicológica. Resulta curioso observar como buena parte de este entrenamiento se viene ya realizando en los centros de estudio, pero de forma solapada y como si estuvieran al margen del plan de estudios, que oficialmente continúa siendo la columna ver- 
tebral de la formación. Becas, intercambios, viajes, redes y prácticas en centros ajenos, adquieren en la actualidad una importancia desproporcionada bajo el punto de vista del "plan de estudios" de una ciencia lineal y acumulativa, pero todavía resultan insuficientes desde la perspectiva que una sociedad actual tiene del conocimiento.

\section{PSICOLOGIA SANITARIA Y PSICOLOGÍA SOLIDARIA}

Otro condicionante de la concepción acumulativa del conocimiento es la organización jerárquica de las disciplinas que componen un área de estudio, una especie de árbol lógico por el que hay que ascender ordenadamente. Primero hay que estudiar esto y lo otro, en segundo curso ya se puede estudiar aquello $y$, en el último eslabón, ya se tiene el conocimiento adecuado. A su vez, las disciplinas que componen el árbol tienden a convertirse en especialidades, porque los docentes también quieren tener aplicaciones en el resto del bosque. Entonces aparecen los gremios cerrados donde se defienden las fronteras por encima de todo, junto con los símbolos de identidad, ya sea una bata blanca, un laboratorio o un computador. El resultado final es una psicología con visión de artrópodo, donde se representa el campo visual como un mosaico de disciplinas y especialidades, hasta el punto de convencerse de que en eso consiste la realidad psicológica.

No se puede dudar de que la ordenación del conocimiento pueda tener cierta utilidad didáctica, ni que las especialidades faciliten la integración profesional, pero es un error convertir una estrategia en una militancia. Sin embargo, la mayor equivocación es pensar que la sociedad actual percibe la actividad psicológica con los mismos ojos que los académicos o los gremios profesionales, porque hay que reconocer la dificultad social que entraña diferenciar entre psicología clínica, psicopatologia, psicología de la salud, psicodiagnóstico y otras parecidas o, por recorrer todo el espectro, entre psicología social, política, tráfico, atención en catástrofes y demás, aunque entiendo perfectamente que esta indiferencia escolástica produce escalofríos entre los académicos creyentes. A riesgo de ser tachado de ignorante, me atrevo a señalar que toda 
la psicología, desde el punto de vista de la sociedad de hoy, se divide principalmente en dos grandes regiones, la psicología sanitaria y la psicología solidaria, al margen de reconocer que no son ni exhaustivas ni excluyentes, pero desde luego son las más reconocidas desde el punto de vista del observador.

\section{a) Psicología sanitarla}

En cuanto a la psicología sanitaria, tiene un largo pasado y un presente especialmente original que no es posible recorrer aquí en toda su profundidad, pero merece la pena dibujar alguna pincelada para darle sentido al argumento. Las relaciones de la psicología con la medicina o, si se prefiere, con la psiquiatría son muy antiguas y están cargadas de ambivalencias. Como ya mencioné en otro escrito, una gran parte de los que se consideran fundadores de la psicología cientifica tenían una sólida formación médica, además de otros intereses casi siempre relacionados con la filosofía. Basta recordar, por ejemplo, que los iniciadores de la psicofísica habían estudiado medicina, Ernst H. Weber en Heidelberg y Gustav Th. Fechner en Leipzig. También Wilhelm M. Wundt, siempre mencionado por establecer el primer laboratorio de psicología experimental, era médico por Heidelberg. La lista de médicos en estos orígenes de la psicología científica y durante bastante tiempo después es suficientemente larga como para sospechar que no podían estar alejados por completo de las preocupaciones patológicas, las alteraciones funcionales o los comportamientos anormales. Es cierto que la psicología experimental, en sus inicios, perseguia la mente del hombre normal, pero al menos siempre tuvo en cuenta que las anomalías, tanto en los contenidos como en los procesos, eran una fuente importante de contraste e investigación de la normalidad.

Sin embargo, surge algo nuevo hacia la mitad del siglo pasado, cuando a partir de la Segunda Guerra aparece una nueva sociedad con características distintas y necesidades aceleradas de una población que plantea fuertes exigencias sociales sobre la base de un fuerte desarrolio económico. La psicología sanitaria de entonces, a través de nombres y disciplinas diversas, es el resultado de las 
aspiraciones en expansión de esa sociedad que confía en la ciencia y espera resultados.

A partir de los años setenta, se generaliza la formación de profesionales y los estudios especializados. Comienza ahora la construcción social de la salud mental, caracterizada por implicar a toda la sociedad y a su dinámica interactiva en la explicación de la salud y la enfermedad de los individuos y los grupos afectados, por la desconfianza hacia los viejos diagnósticos y etiologías, y por la exigencia de aplicaciones sociales inmediatas.

Los últimos veinte años del pasado siglo llevan hasta las últimas consecuencias el razonamiento de que, si la mayor parte de la salud y de la enfermedad mental es una construcción social en la que participamos todos, entonces tampoco es necesario dividir con demasiado énfasis la salud física y la mental, porque nuestro desarrollo, bienestar y satisfacción dependen principalmente de factores biopsicosociales. La psicología sanitaria tiende a ocuparse ahora del conjunto de estos ámbitos, al servicio del valor fundamental de la salud, con la principal finalidad de aplicaciones concretas en los sectores de mayor urgencia social.

Una gran parte de los psicólogos actuales reivindican su presencia en todo tipo de instituciones sanitanas y también es cierto que la mayor parte de la sociedad los percibe desde ese punto de vista. En ningún momento de su historia han tenido tanta actividad, investigación y potencia profesional como en los últimos tiempos. Puede plantearse la eficacia, fundamentos y comprobación de modelos concretos, pero es indiscutible que, en general, está consiguiendo una sensibilidad social y una atención de todo tipo de instituciones sociales como nunca había tenido, con todo lo que esto significa de beneficios para todas las personas de la sociedad actual.

\section{b) Psicología solldarla}

No toda la psicología reivindica su carácter terapéutico a través de las instituciones sanitarias, la otra cara de la moneda representa lo que entiendo aquí por psicología solidaria. También tiene una larga tradición que fácilmente se puede remontar a los Ideólogos 
franceses de la época de la Revolución, una especie de psicólogos empeñados en realizar un estudio científico de las ideas, cuya mayor preocupación consistía en facilitar a sus contemporáneos una adaptación soportable ante el nuevo orden que se estaba produciendo. La "ldeología” como una actividad solidaria hacia los ciudadanos ante los cambios revolucionarios que se estaban produciendo en todos los órdenes de la vida, constituye sin duda un antecedente claro del otro papel que la psicologia, utilizando múltiples nombres y disciplinas, ha desempeñado posteriormente de forma más o menos sistemática.

Al igual que ocurre a partir de la Revolución Francesa con el impulso solidario del nuevo conocimiento social, el caso de los Estados Unidos es todavia más claro en cuanto a su relación entre los planteamientos sociales iniciales y la preocupación por el bienestar individual y colectivo. El origen de la mayor parte de sus universidades, departamentos de sociología y economía o de psicología social, se debe al esfuerzo de los reformadores sociales, casi siempre de inspiración religiosa, preocupados por los problemas de la inmigración pobre, la marginación social o el contacto y los prejuicios entre razas y culturas distintas. Bajo este punto de vista, más que buscar ejemplos concretos hay que hablar de una trayectoria continua de preocupación por el bienestar y la adaptación de las personas. Desde la defensa del grupo primario frente a la conflictividad de la vida urbana de Charles Horton Cooley o los estudios sobre las actitudes y el desarraigo de los inmigrantes polacos de W. I. Thomas, pasando por los estudios sociológicos sobre la ciudad y sus conflictos mediante una observación participante de Robert Park, hasta llegar a la dinámica de grupos de Kurt Lewin como una especie de terapia social democrática para la resolución de conflictos sociales, étnicos y personales, casi todo el pensamiento social americano se enfoca hacia el bienestar solidario de los individuos y grupos.

Es cierto que toda esta corriente de psicología solidaria se desarrolla de forma bastante diferente en la época actual, en función de los valores de la comunicación, el consenso y la participación, y también en virtud de las distintas tradiciones culturales, ya sean principalmente protestantes o católicas. Mientras que la psicología solidaria en Estados Unidos se desarrolla especialmente a través de 
los nuevos movimientos sociales, entre nosotros prosperan las organizaciones no gubernamentales, casi siempre de inspiración religiosa, o los organismos que envían grupos de psicólogos como apoyo en las catástrofes o tragedias colectivas. Sea como sea, la psicología solidaria es la otra cara de la psicología ante la sociedad de nuestro tiempo, maquillada con innumerables nombres técnicos especializados como psicología de género, política, organizacional, de seguridad vial o ambiental.

Cuantas más disciplinas y especialidades aparecen, menos se diferencian entre sí y con mayor razón necesitan negociar sus fronteras mediante pactos transdisciplinares. Pero a la sociedad le preocupa poco estas disquisiciones académicas y solo entiende la psicología como una ayuda a sus problemas, que casi siempre provienen del amplio mundo de la salud o de la incapacidad de actuación unitaria en los grupos sociales.

\section{CONCLUSIÓN}

¿Qué es lo que debe saber un psicólogo y qué debe saber un profesor de psicología? Pues depende, todo depende de aquello que la sociedad le pida a ese psicólogo o a ese docente. ¿Qué es lo que va a hacer ese psicólogo $y$, en consecuencia, a enseñar ese profesor? Porque no es lo mismo que trabaje en una unidad de cuidados intensivos o en el diseño de los paneles de tráfico, que participe en una organización humanitaria o en una campaña electoral, por poner ejemplos al azar. El supuesto de que la psicología es un conjunto organizado de conocimientos y que, una vez alcanzado, se puede aplicar a cualquier campo, pertenece a tiempos anteriores y dificilmente se puede mantener hoy en día.

A medida que se construyen más planes de estudio, nos ìremos dando cuenta de que no es posible un programa conjunto para todo tipo de psicólogos, porque el conocimiento compartido es muy escaso y las necesidades profesionales cada vez más amplias. Eso conduce a una preparación diferenciada, que no es fruto de la especialización sino de la integración social en una actividad profesional. Dicho de otra forma, los psicólogos comenzaron diferenciándose por escue- 
las, tendencias y orientaciones teóricas, mientras que ahora se distinguen por lo que tienen que hacer en la sociedad.

Hay psicólogos y psicólogos, y ninguno sabe más que el otro o debe tener una preparación más o menos extensa, simplemente tiene que estar preparado para comunicarse y participar dentro de su grupo profesional y saber hacer lo que está consensuado con la sociedad. Ahora se entenderá mejor que no existe cuadro sinóptico que pueda abarcar los estudios de tantos y tan variados psicólogos.

Comienza a ser habitual que una empresa determinada le pida a la universidad o a algún centro de enseñanza que le prepare un pequeño grupo de profesionales para realizar una actividad específica. Es la enseñanza a la carta, donde el gusto del consumidor viene determinado por las exigencias sociales. Entre el cuadro sinóptico y el menú han transcurrido más de cien años para la psicología y, sin embargo, buena parte de los planes de estudio actuales continúan confundiendo lo uno con lo otro.

En todo caso, esto no es más que una opinión y seguramente no es la más generalizada, ni tampoco tiene pretensiones de serlo. Pero eso solo demuestra lo que venimos comentando, que hay psicólogos y psicólogos, y todos pueden tener un papel relevante en esta sociedad que cada día necesita más de todo. 